\title{
Evaluation of 5G Key Technique: Non-Orthogonal Multiple Access
}

\author{
Haiyang Yu, Jaeho Choi
}

\begin{abstract}
Non-orthogonal multiple access has been put forward as a key technique for $5 G$. It can provide power-domain and code-domain multiplexing and enables to satisfy the data demand. Its capacity and spectral efficiency are investigated-ed and analyzed. In comparison to the conventional orthogonal multiple access, the existing dominant non-orthogonal multiple access can provide a higher quality data service for multiple users when the transmitted signals are empowered by the power allocation technique and the received signals are decoded by the channel interference cancellation scheme. In this study, NOMA is found to be a front-edge technology the $5 G$ communications.
\end{abstract}

Index Terms: Non-orthogonal multiple access, orthogonal multiple access.

\section{INTRODUCTION}

Recently, a novel multiple access scheme named non-orthogonal multiple access (NOMA) has been considered highly in the communications society and its useful-ness is studied to satisfy the data demand required for the next generation communication systems [1-5]. These literatures present advantages of the NOMA over the orthogonal multiple access (OMA) by providing several key evidences of NOMA's superior capabilities in terms of spectral efficiency and balanced user fairness. In contrast to conventional OMA, the existing dominant NOMA scheme can provide power-domain and code-domain multiplexing. At the transmitter, the transmission signals are superposed by using superposition coding; at the receiver the received signals are decoupled by using the successive interference cancellation (SIC). Furthermore, when superimposing signals at the transmitter (or at the base station) a difference power allocation scheme can be used in order to improve the spectral efficiency of the system; hence, NOMA can support a higher number of users where multiple users are cooperating each other to receive their own signals and to relay the signals intended for their neighboring users in cooperation. In [2], the authors derived analytical expressions of the average and optimum sum rate to show that NOMA outperforms the conventional OMA. In addition, the authors in [3] have investigated the advantages of NOMA in supporting complex connectivity and also meeting different quality of service requirements of next generation communications systems such as 5G. In [6] and [7], the communications security issue has been considered at the physical layer that NOMA is capable of providing and secure and reliable communications over the physical transmission

Revised Version Manuscript Received on August 19, 2019.

Haiyang Yu, Department of Electronic Engineering, CAIIT, Chonbuk National University, Chonju, Korea, 561-756.

Jaeho Choi, Department of Electronic Engineering, CAIIT, Chonbuk National University, Chonju, Korea, 561-756. channel. In [8] and [9], the impact of relay selection on the performance of NOMA is performed to demonstrate that a two-stage RS strategy can achieve the minimal outage probability among all possible RS schemes and realize the maximal diversity gain. In [10] and [11], the authors proposed multicast beamforming with superposition coding for NOMA for supporting multiple users as two-stage beamforming methods and formulated a minimum total transmission power beamforming problem to find beamforming vectors and powers for the near and far users.

\section{PROPERTIES OF OMA AND NOMA}

Figure 1 shows the capacity comparison between NOMA and OMA. The figure on the left is the cases of non-symmetric capacities provided for two users R1 and R2; the figure on the right is the ones for symmetric capacities. In both cases, the total capacity of OMA is always smaller than that of the NOMA. Furthermore, the capacity of NOMA can approach the upper bound of the total channel capacity while OMA can only achieve sub-optimal. Since OMA can reach the capacity bound at the fixed number of points, the user fairness may not always reach a reasonable level when OMA is used while the NOMA can successfully provide reasonable user fairness between the multiple users. Particularly, for the two-user systems as shown in Figure 3, the capacities for two users in OMA are $\frac{1}{2} \log _{2}\left(1+G\left|k_{1}\right|^{2}\right)$ and $\frac{1}{2} \log _{2}\left(1+G\left|k_{2}\right|^{2}\right)$, respectively, while the capacities for two users in NOMA are equal to $\log _{2}\left(G\left|k_{2}\right|^{2}\right)$. In other words, it is obvious to find that the total capacity of NOMA is larger than that of the OMA as the coefficient of 0.5 in the capacities of OMA users affects so much for the sum.

In practice, the users are not normally in the different channel conditions and their individual maximum achievable data rates become non-symmetric. In such cases, the non-symmetric power allocation (NSPA) scheme can be used during transmission signal superposition. More specifically, in NSPA, assuming that the channel information is available, by allocating a stronger power to the signals intended to the user sitting in a better channel condition. The other aspect of NSPA concerns with the user fairness. Whenever NSPA is used, the user fairness is hampered because of the fact that a larger part of total transmission power is used for a particular user. Hence, the trade-off between power allocation and user fairness is a very important issue in the next generation

Published By

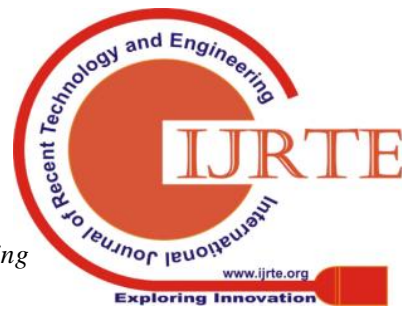


communications systems. In that aspect the NOMA is a better choice that the OMA because NOMA can do better job improving the total achievable channel capacity of the system using the NSPA and it can also do a good job for maintaining

Table I. Comparison of OMA and NOMA

\begin{tabular}{|c|c|c|}
\hline Transmission & Advantages & Disadvantages \\
\hline \multirow{4}{*}{ OMA } & Easier & $\begin{array}{c}\text { Low spectral } \\
\text { efficiency }\end{array}$ \\
\cline { 3 - 3 } & detection & $\begin{array}{c}\text { Less number of } \\
\text { users }\end{array}$ \\
\cline { 3 - 3 } & bad user fairness \\
\hline \multirow{4}{*}{ NOMA } & $\begin{array}{c}\text { High } \\
\text { spectral } \\
\text { efficiency }\end{array}$ & $\begin{array}{c}\text { More complex in } \\
\text { receiver side }\end{array}$ \\
\cline { 2 - 3 } & $\begin{array}{c}\text { Better user } \\
\text { fairness }\end{array}$ & $\begin{array}{c}\text { Higher sensitivity to } \\
\text { channel uncertainty }\end{array}$ \\
\cline { 2 - 3 } & Low latency & \\
\cline { 2 - 3 } & $\begin{array}{c}\text { High } \\
\text { connection } \\
\text { density }\end{array}$ & \\
\cline { 2 - 3 } & $\begin{array}{c}\text { Support } \\
\text { diverse QoS }\end{array}$ & \\
\hline
\end{tabular}

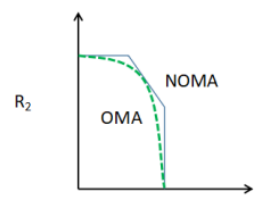

$\mathrm{R}_{1}$

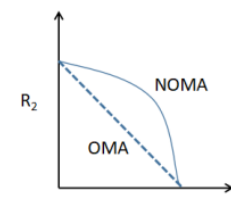

$\mathrm{R}_{1}$
Fig. 1. Capacity comparison between NOMA and OMA

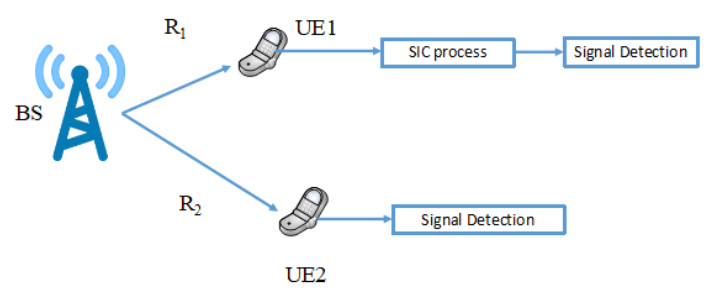

Fig. 2. Illustration of working process for cooperative NOMA

On the other hand, as far as the bandwidth is concerned, the NOMA is also a better choice than the OMA. While OMA needs number of orthogonal sub-channels to carry the data, NOMA needs single channel. OMA works efficiently when the channel orthogonality is guaranteed; however, it requires much overhead. OMA also can overcome the channel distortion with a simpler mechanism such as the single-tap equalizer; however, it also needs multi-user interference cancellation mechanism similar to the SIC in NOMA. Overall, the complexities in transmission and also in reception in both technologies requires more careful investigation, though, NOMA has a lower complexity and it is also capable of slower degradation in performance as the channel condition gets worse. the user fairness.

\section{COOPERATIVE NOMA TRANSMISSION}

In Figure 2, it shows a cooperative NOMA (C-NOMA) system where the base station is transmitting the superposed signal to two users using power-domain and code-domain multiplexing [12]. The multiple users, channel information, power allocation, relay, and signal inference cancellation are the main elements of the C-NOMA system. From BS transmission to multiple user reception, it follows:

1. The BS needs to obtain the channel information for each of two users.

2. The power is allocated to the signals for two users based on their channel information and service priority and requirement. The larger portion of the transmission power is allocated to the user sitting in the worse channel condition or to the user with a higher service requirement. In Figure, the user UE1 is selected as a node needing a priority service; the BS allocates the more power to the UE1 signal. After power allocation by the base station, the signals for two users are superimposed and sent simultaneously in the first time slot.

3. Upon receiving the signal from $\mathrm{BS}$, UE1 decodes its message from the superimposed signal by following the principle of SIC. During the second time slot, assume that UE1 can decode two signals, sends the same superimposed signal to UE2. Upon receiving the superimposed signal, UE2 decodes its own message

This C-NOMA can be used for local short-range communications techniques like ultra-wideband and Bluetooth. In [13], the authors explain how the cooperative NOMA outperforms non-cooperative NOMA in terms of outage probability and the channel capacity, in which the cooperative NOMA achieves a greater diversity gain for multiple users.

On the other hand, some literatures consider the short-comings of the cooperative NOMA systems such as maximum system overhead in multi-user system, time waste, and increased complexity. Solutions have been proposed to solve the problems [14].

\section{ANALYTICAL EVALUATION FOR NOMA AND OMA RESULTS}

The analytical simulation is performed for NOMA and OMA and the results are compared in terms of CDF. The $\mathrm{CDF}$ increases to the maximum as the number of multiple users increase; the OMA saturates earlier with the smaller number of users than that the NOMA.

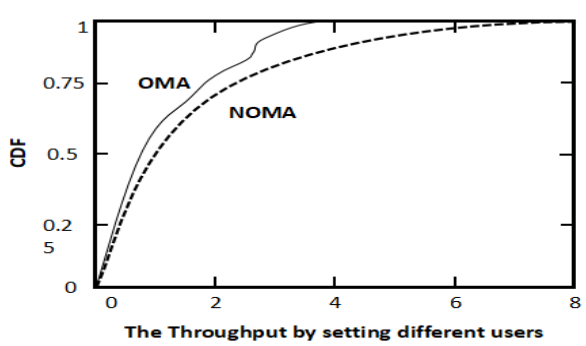

Fig. 3. The comparison of NOMA and OMA in terms of CDF 


\section{CONCLUSION}

We have discussed the advantages of NOMA and also compared the performance of NOMA and OMA. NOMA performs better than OMA yielding a higher throughput by exploiting power-domain and code-domain multiplexing. Furthermore, the cooperative NOMA can improve the capacity of the system better than the conventional NOMA. This advantageous fact of NOMA fits to the needs of $5 \mathrm{G}$ technology, which calls for QoS and a higher connectivity. In fact, the recent development in $5 \mathrm{G}$ finds a good possibility of NOMA for getting a significant improvement in the throughput of the communication systems.

\section{ACKNOWLEDGEMENTS}

This work was supported partly by funds provided by BK21+ and Chonbuk National University of Korea.

\section{REFERENCES}

1. T. Cover, "Broadcast channels," IEEE Trans. Inf. Theory, vol. IT-18, no. 1, pp. 2-14, 1972.

2. Z. Chen, Z. Ding, X. Dai, and R. Zhang, "An optimization perspective of the superiority of NOMA compared to conventional OMA," to be appeared in IEEE Trans. Signal Proc., 2017.

3. Z. Ding, Z. Yang, P. Fan, and H. V. Poor, "On the performance of non-orthogonal multiple access in $5 \mathrm{G}$ systems with randomly deployed users, " IEEE Signal Process. Lett., vol. 21, no. 12, pp. 1501-1505, 2014.

4. Z. Wei, J. Yuan, D. W. K. Ng, M. Elkashlan, and Z. Ding, "A survey of downlink non-orthogonal multiple access for $5 \mathrm{G}$ wireless communication networks," ZTE Comm., vol. 14, no. 4, pp. 17-26, 2016.

5. L. Dai, B. Wang, Y. Yuan, S. Han, C.-L. I, and Z. Wang, "Nonorthogonal multiple access for 5G: Solutions, challenges, opportunities, and future research trends," IEEE Comm. Mag., vol. 53, no. 9, pp. 74-81, 2015.

6. B. He, A. Liu, N. Yang, and V. K. Lau, "On the design of secure nonorthogonal multiple access systems," arXiv preprint arXiv:1612.06961, 2016.

7. L. Fan, X. Lei, N. Yang, T. Q. Duong, and G. K. Karagiannidis, "Secure multiple amplify-and-forward relaying with cochannel interference," IEEE J. Sel. Topics Signal Process., vol. 10, no. 8, pp. 1494-1505, 2016.

8. Z. Ding, H. Dai, and H. V. Poor, "Relay selection for cooperative NOMA," IEEE Wireless Comm. Lett., vol. 5, no. 4, pp. 416-419, 2016.

9. J. B. Kim, M. S. Song, and I.-H. Lee, “Achievable rate of best relay selection for non-orthogonal multiple access-based cooperative relaying systems," in Proc. Int. Conf. Inf. Comm. Tech. Conv. (ICTC), pp. 960-962, 2016.

10. J. Choi, "Minimum power multicast beamforming with superposition coding for multiresolution broadcast and application to NOMA systems," IEEE Trans. Comm., vol. 63, no. 3, pp. 791-800, 2015.

11. M. Al-Imari et al., "Uplink Nonorthogonal Multiple Access for 5G Wireless Networks," Proc. 11th Int'l. Symp. Wireless Comm. Sys., pp. 781-85, 2014.

12. A. S. Ibrahim, A. K. Sadek, W. Su, and K. J. R. Liu, "Cooperative communications with relay-selection: When to cooperate and whom to cooperate with" IEEE Trans. Wireless Comm., vol. 7, no. 7, pp. 2814-2827, 2008.

13. Z. Ding, M. Peng, and H. V. Poor, "Cooperative Non-Orthogonal Multiple Access in 5G Systems," IEEE Comm. Lett., vol. 19, no. 8, pp. 1462-65, 2015.
14. Z. Ding, P. Fan, and H. V. Poor, "Impact of User Pairing on 5G Non-Orthogonal Multiple Access," IEEE Trans. Veh. Tech., vol. 65, no. 8, pp. 6010-23, 2016. 